\title{
OPTIMIZATION OF CHEMICAL PROCESSES UNDER UNCERTAINTY
}

\author{
G. M. Ostrovsky \\ Department of Chemical Engineering, U-222 \\ University of Connecticut \\ 191 Auditorium Road, Storrs, CT 06269, USA. \\ ostrovsk@engr.uconn.edu
}

\author{
L. E. K. Achenie \\ As above \\ achenie@engr.uconn.edu
}

Yu. M. Volin

Karpov Institute of Physical Chemistry

Vorontsovo Pole 10

Moscow, 103064, Russia.

volin@cc.nifhi.ac.ru

\begin{abstract}
The problem of determining optimal equipment sizes and regimes of chemical processes that guarantee flexibility of the processes under uncertainty is considered in this paper. The effect of multiextremality of the solution is investigated. A comparative analysis of deterministic methods available in the open literature has been carried out as a result. All the methods require some convexity (or concavity) assumptions, which are difficult to verify for practical problems. To address the latter, we have developed algorithms with minimal convexity (or concavity) requirements for this problem.
\end{abstract}

Keywords: Flexibility Analysis, Uncertainty Analysis, Chemical Process Design, Nonlinear Programming

\section{INTRODUCTION}

In chemical process (CP) design some design specifications such as (a) safety, (b) ecological, and (c) performance specifications must always be

The original version of this chapter was revised: The copyright line was incorrect. This has been corrected. The Erratum to this chapter is available at DOI: 10.1007/978-0-387-35514-6_15

M.J.D. Powell and S. Scholtes (Eds.), System Modelling and Optimization: Methods, Theory and Applications. (O) 2000 IFIP International Federation for Information Processing.

Published by Kluwer Academic Publishers. All rights reserved. 
met. Often such specifications are formulated either as soft or as hard constraints, with the presumption that a violation of hard constraints is not allowed. In this paper, we confine ourselves to hard constraints only.

The satisfaction of design specifications is complicated by the presence of uncertainties in design models, such as

- inherent inaccuracies of coefficients in the mathematical models,

- changes in some of the coefficients in the mathematical models during the $\mathrm{CP}$ operation (for example rate constants, heat and mass transfer coefficients),

- variations in some of the parameters (e.g. temperature, flow rates, species concentrations) associated with external streams during the CP operation.

It is of critical importance for a safe and economic operation of the process to account for the uncertainties discussed above when the optimal design structure, equipment sizes and regimes of the $\mathrm{CP}$ operation subject to design specification are determined. Several authors have addressed aspects of optimal design under uncertainty in the recent literature (Biegler, Grossmann and Westerberg, 1997), (Halemane and Grossmann 1983), (Grossmann and Floudas, 1987), (Pistikopoulos and Grossmann, 1989), (Pistikopoulos and Ieraptritou, 1995). Many contributions in the operation literature, however, rely on the availability of probability distribution functions for characterizing parametric uncertainties. Since practicing engineers very rarely know these distributions (without significant guess work), we believe it is important to consider ways of dealing with uncertainty which do not require probability distributions to be known. Halemane and Grossmann (1983) developed two formulations for analyzing the optimal design problem under uncertainty. The first formulation is an evaluation of the CP flexibility (its ability to satisfy process specifications during the operation stage), which has the form

$$
F_{1}(d) \leq 0
$$

where the flexibility function $F_{1}(d)$ is of the form

$$
F_{1}(d)=\max _{t \in T} \min _{z \in Z} \max _{j \in J} g_{j}(d, z, t) .
$$

Here, $J=\{1, \ldots, m\}, d$ is a vector of design variables, $z$ is a vector of control variables, $Z$ is a region of admissible values of control variables, 
and

$$
T=\{t: \bar{t} \leq t \leq \overline{\bar{t}}\}
$$

is the domain for the uncertain parameters. The reduced process constraints

$$
g_{j}(d, z, t) \leq 0, \quad j=1, \ldots, m
$$

are obtained from the original mathematical model

$$
\begin{aligned}
& \varphi(d, x, z, t)=0 \\
& \bar{g}(d, x, z, t) \leq 0
\end{aligned}
$$

by explicitly solving for the vector of state variables $x$ which has the same dimension as $\varphi$. Equations (4) are state equations (i.e. material and heat balance equations), while inequalities (5) are design specifications.

$F_{1}(d)$ can be represented in the form

$$
F_{1}(d)=\max _{t \in T} h(d, t)
$$

where

$$
h(d, t)=\min _{z \in Z} \max _{j \in J} g_{j}(d, z, t)
$$

Also

$$
\begin{aligned}
h(d, t) & =\min _{z \in Z, v} v \\
g_{j}(d, z, t) & \leq v, \quad j=1, \ldots, m
\end{aligned}
$$

Therefore, the calculation of the flexibility function $F_{1}(d)$ is reduced to the maximization of $h(d, t)$ with respect to $t$. Halemane and Grossmann (1983) have shown that, in general, $h(d, t)$ is multiextremal and nondifferentiable.

The second formulation (of Grossmann et. al.) is the two-stage optimization problem under uncertainty (TSOP), which has the form

$$
\begin{aligned}
f_{1} & =\min _{d \in D} E\left[f^{*}(d, t)\right] \\
F_{1}(d) & \leq 0
\end{aligned}
$$

where $D$ is a region of admissible values of the vector $d, E\left[f^{*}(d, t)\right]$ is the mathematical expectation of $f^{*}(d, t)$ with respect to $t$ with $f^{*}(d, t)$ given by 


$$
\begin{aligned}
f^{*}(d, t) & =\min _{z \in Z} f(d, z, t) \\
g_{j}(d, z, t) & \leq 0, \quad j=1, \ldots, m .
\end{aligned}
$$

Here $f(d, z, t)$ is the objective function in the original optimization problem.

Using Gaussian quadrature (Carnahan, 1969) to approximate the multiple integral in the objective function, one can reduce the latter problem to the following problem (Halemane and Grossmann, 1983)

$$
\begin{aligned}
f_{1} & =\min _{z^{i}, d \in D} \sum_{i \in I_{1}} w_{i} f\left(d, z^{i}, t^{i}\right) \\
g_{j}\left(d, z^{i}, t^{i}\right) & \leq 0, \quad j \in I_{1}, \\
F_{1}(d) & \leq 0,
\end{aligned}
$$

where $w_{i}$ are weights, $t^{i}$ is an approximation point, $z^{i}$ is a vector of control variables associated with the point $t^{i}$, and $I_{1}$ is a set of indices of the approximation points. Notice that the distribution functions which are required for the calculation of the mathematical expectation are often unknown. In this case the weights and approximation points must be selected using engineering insight. Similar problems arise in the design of other technical systems such as electrical circuits. Conceptually, the TSOP (9) determines the optimal design margins that guarantee satisfaction of process specifications under uncertainty, assuming the given uncertainty bounds are correct. This guarantee, however, can only be given if inequality $F_{1}(d) \leq 0$ holds for the global solution of the flexibility problem (6). Therefore, it is appropriate that we discuss different deterministic methods and the conditions under which the global solution of (6) is achieved.

\section{COMPARATIVE ANALYSIS OF CURRENT METHODS}

In this section, we compare current methods for estimation of process flexibility and optimization of chemical processes under uncertainty. Specifically, we limit ourselves to the following methods: (i) the method of Halemane and Grossmann (1983) (the HG method), (ii) the active constraint sets method (the ACS method of Grossmann and Floudas, 1987 and Pistikopoulos and Grossmann, 1989), (iii) the SG method of Swaney and Grossmann (1985), and (iv) the upper and lower bounds method (the ULB method of Ostrovsky and Volin, 1994, 1997). These methods are fairly representative of the research effort documented in the open literature, and they all employ local optimization methods. 
Let us consider the first method. Swaney and Grossmann (1985) have shown that the global solution of the flexibility problem in (6) is obtained at a vertex of the parameter set $T$ if the following condition is met

Condition 1: The functions $g_{j}(d, z, t)$ are jointly quasi-convex in $z$ and $d$. In addition they are one-dimensional quasi-convex in $t$.

We note that a function $f(x)$ is one-dimensional quasi-convex in $x$ if it is quasi-convex separately with respect to each component of $x$. The search for the maximum of $h(d, t)$ in $T$ can now be reduced to a search among the vertices of $T$ (Halemane and Grossmann, 1983). The computational effort of the method is, in general, proportional to the number of vertices of $T, 2^{r}$, where $r$ is a dimension of the vector $t$. Swaney and Grossmann (1985) introduced the flexibility index, which characterizes the largest uncertainty region that the design can handle for feasible operation. For the case when each $g_{j}(d, z, t)$ is monotonic is $t$, Swaney and Grossmann (1985) proposed an algorithm for the flexibility index problem which uses a branch and bound (BB) strategy to search among the vertices of $T$. Kabatek and Swaney (1992) suggested a modification, which permits to find non-vertex solutions; however, the procedure does not guarantee a global solution.

We next consider the ACS method. Under certain conditions, the number of active constraints in problem (8) is equal to $q+1$, where $q$ is the number of control variables. Based on this, Grossmann and Floudas (1987) suggested a method for solving (2) as follows:

1 Identify all potentially active constraint sets $A S(k)$ ( $k$ is the index of the set) consisting of $q+1$ constraints. Let us denote the number of such sets as $n_{A S}$.

2 Solve the problem

$$
\begin{aligned}
u^{k} & =\max _{t, z} u \\
g_{j}(d, z, t) & =u, \quad j \in A S(k)
\end{aligned}
$$

for all $k=1, \ldots, n_{A S}$.

3 Determine

$$
F_{1}=\max _{k} u^{k}
$$

Pistikopoulos and Grossmann (1989) generalized the method for the retrofit design problem. Grossmann and Floudas (1987) showed that the 
ACS method gives the global solution of problem (2) under satisfaction of the following:

\section{Condition 2:}

1 Functions $g_{j}(d, z, t)$ are strictly quasi-convex in $z$ for fixed $t$

2 Functions $g_{j}(d, z, t)$ are jointly quasi-concave in $z$ and $t$.

One can show that if Condition 2.1 is not satisfied, the ACS method does not guarantee a local or global solution. The alternative ULB method (Ostrovsky, Volin et. al., 1994, 1997) uses the branch and bound strategy for the calculation of $F_{1}$. It seeks the maximum of $h(d, t)$ over a partitioning of the region $T$ into subregions $T_{i}$ and is based on the inequality (Ostrovsky, Volin et. al., 1994)

$$
F_{2 i} \geq h(d, t), \quad \forall t \in T_{i},
$$

where

$$
F_{2 i}=\min _{z \in Z} \max _{j \in J} \max _{t \in T_{i}} g_{j}(d, z, t) .
$$

Consequently one can use $F_{2 i}$ as an upper bound of $h(d, t)$ on $T_{i} . F_{2 i}$ can be evaluated by solving the problem

$$
\begin{aligned}
F_{2 i} & =\min _{z \in Z, u} u \\
\max _{t \in T_{i}} g_{j}(d, z, t) & \leq u, \quad j=1, \ldots, m .
\end{aligned}
$$

The ULB method for solving the TSOP is a two-level iterative procedure, which employs a partitioning of $T$. The upper level serves to partition $T$ using information obtained from the lower level. At iteration $k$ of the upper level the lower level is used to calculate an upper bound $f^{U,(k)}$ and a lower bound $f^{L,(k)}$ of $f$. Suppose at iteration $k$ of the upper level the set $T$ is partitioned into $N_{k}$ subregions $T_{l}, l=1, \ldots, N_{k}$. On each $T_{l}$ the algorithm will automatically update a set of critical points: $S_{2, l}^{(k)}=\left\{\theta^{i}: \theta^{i} \in T_{l}, i \in I_{l}\right\}$ where $I_{l}$ is the index set of critical points on the subregion $T_{l}$. The upper bound $f^{U,(k)}$ for a new set of subregions is calculated by solving the problem

$$
\begin{aligned}
f^{U,(k)} & =\min _{d, z^{i}, z^{l}} \sum_{i \in I_{1}} w_{i} f\left(d, z^{i}, t^{i}\right) \\
g_{j}\left(d, z^{i}, t^{i}\right) & \leq 0, \quad i \in I_{1}, \quad j=1, \ldots, m,
\end{aligned}
$$




$$
\max _{t \in T_{l}} g_{j}\left(d, z^{l}, t\right) \leq 0, \quad l=1, \ldots, N_{k}, \quad j=1, \ldots, m
$$

where $z^{l}$ is a control variables vector corresponding to the subregion $T_{l}$ and $I_{1}$ is the set of approximation points (see (9)). The lower bound $f^{L,(k)}$ is calculated at the lower level as

$$
\begin{aligned}
f^{L,(k)} & =\min _{d, z^{i}, z^{l}} \sum_{i \in I_{1}} w_{i} f\left(d, z^{i}, t^{i}\right) \\
g_{j}\left(d, z^{i}, t^{i}\right) & \leq 0, \quad i \in I_{1}, j=1, \ldots, m, \\
g_{j}\left(d, z^{l q}, t^{l q}\right) & \leq 0, \quad \forall t^{l q} \in T_{l}, l=1, \ldots, N_{k}, \quad j=1, \ldots, m,
\end{aligned}
$$

where $z^{l q}$ is a vector of control variables corresponding to the point $t^{l q}$.

Now consider the upper level. At each iteration a partitioning of $T_{l}$ is performed. The partitioning strategy strongly affects the computational complexity of the procedure. The simplest approach is to partition all subregions. However, the dimension of problems (13) and (15) will be very large. To alleviate this problem, we employ the following heuristic: at the $k$-th iteration, $T_{l}\left(l=1, \ldots, N_{k}\right)$ is partitioned only if for this $l$ the constraints (14) are active for at least one $j,(j=1, \ldots, m)$.

Next, consider the lower level. Problem (15) is a standard nonlinear program that can be handled by standard algorithms. Problem (13), however, is not a conventional NLP. Therefore, a two-level iterative procedure is used for its solution. In the first step we solve the problem

$$
\begin{aligned}
f_{2} & =\min _{d, z^{i}, z^{l}} \sum_{i \in I_{1}} w_{i} f\left(d, z^{i}, t^{i}\right) \\
g_{j}\left(d, z^{i}, t^{i}\right) & \leq 0, \quad i \in I_{1}, \quad j=1, \ldots, m, \\
g_{j}\left(d, z^{l}, t^{l q}\right) & \leq 0, \quad \forall t^{l q} \in S_{2, l}, l=1, \ldots, N_{k}, \quad j=1, \ldots, m .
\end{aligned}
$$

Let $\left[d^{*}, z^{*}\right]$ be the solution to the problem, and let $n$ be the iteration counter at the lower level. We note that in problem (16), the control vector $z^{l}$ is associated with all the critical points of $T_{l}$ whereas in problem (15), there is a control vector $z^{l q}$ for each point $t^{l q}$.

During the second step, a stopping criterion is checked and some of the sets of critical points are extended. For this, the following $m N_{k}$ problems are solved

$$
\max _{t \in T_{l}} g_{j}\left(d^{*}, z^{l *}, t\right), \quad l=1, \ldots, N_{k} ; j=1, \ldots, m .
$$

In the case when it is not possible to obtain an explicit expression for state variables as a function of the variables $[d, z, t]$, problem (17) is 
equivalent to

$$
\begin{aligned}
& \max _{x, t \in T_{l}} \bar{g}_{j}\left(d^{*}, x, z^{l *}, t\right) \\
& \varphi\left(d^{*}, x, z^{l *}, t\right)=0 .
\end{aligned}
$$

For fixed $l$ and $j$, let $t_{j}^{l}$ be the solution of the problem. If the condition

$$
g_{j}\left(d^{*}, z^{*}, t_{j}^{l}\right) \leq 0, \quad l=1, \ldots, N_{k} ; \quad j=1, \ldots, m,
$$

is met, then the solution of problem (13) was obtained. Otherwise those $t_{j}^{l}$ for which conditions (19) are violated are added to the sets of critical points of the corresponding subregions. We will assume that a local optimization method (for example SQP) is used for solving problems (15), (16) and (17). Now we will show that the ULB method gives the global solution of problems (2) and (9) under the assumption of

\section{Condition 3}

1 Functions $g_{j}(d, z, t)$ are jointly quasi-convex in $d$ and $z$ (for problem (2) quasi-convexity in $z$ is sufficient)

2 Functions $g_{j}(d, z, t)$ are quasi-concave in $t$.

3 Functions $f(d, z, t)$ is quasi-convex in $d$ and $z$.

Let us consider problem (2). The ULB method gives the global solution if the upper (see problem (13)) and lower bounds of $h(d, t)$ are global solutions. Rewrite the problem (12) in the form

$$
\begin{aligned}
F_{2 i}(d) & =\min _{z, u} u \\
G_{j}(d, z) & \leq u \\
G_{j}(d, z) & =\max _{t \in T} g_{j}(d, z, t)
\end{aligned}
$$

By Condition 3.2, a local maximum of problem (22) coincides with the global maximum (Bazaraa and Shetty, 1979). One can show that if $g_{j}$ is quasi-convex in $z$ (Condition 3.1), then $G_{j}$ is also quasi-convex in $z$. It follows that the region determined by Eqn. (21) is convex and a local minimum of problem (20) coincides with the global minimum (Bazaraa et. al.,1993). Moreover, the local minimum of problem (8) coincides with its global minimum. Thus, by solving problems (20) and (8), we will obtain the global solutions, i.e., the valid upper and lower bounds for subregion $T$. Therefore, the branch and bound procedure must give the global solution. Similarly, one can show that the local minimum of problem (9) coincides with the global minimum if Condition 3 is satisfied. 
If Condition 3.2 holds, which is the case in many applications, but Condition 3.1 is not satisfied then our procedure only guarantees an upper bound for $F_{1}$. Indeed, in this case we obtain a local minimum $\tilde{F}_{2 i, l o c}$ of the problem (20) associated with problem (2) and since $\tilde{F}_{2 i, l o c} \geq$ $\tilde{F}_{2 i} \geq F_{2 i}$ our upper bound estimate is less tight.

Let us now consider two scenarios for the TSOP. If, on the one hand, the ULB method yields a solution then this solution corresponds to the global solution of problem (17), since we have assumed that Condition 3.2 is satisfied. Hence, by (19), we can guarantee the flexibility of the chemical process (CP). However, since the solution is in general a local minimum of problem (9), the design, although flexible may not be the best.

The second scenario corresponds to the case when the ULB method cannot obtain a solution. This means that the following condition is met

$$
\max _{t \in T} \min _{z \in Z} \max _{j \in J} g_{j}(d, z, t) \geq 0 \quad \forall d
$$

This can result from one of the following: (a) the flexibility of the CP cannot be guaranteed or (b) the solution corresponds to a local minimum of $\tilde{F}_{2}$.

Comparison of several methods with respect to their ability to obtain a global solution and their computational complexity leads us to conclude that they all supplement each other to some extent. Subsequently we recommend the following:

1 if Condition 1 is met and the dimension of the vector $t$ is small, the HG method should be employed;

2 if the dimension of $t$ is not small and each $g_{j}$ is monotonic, the SG method is appropriate;

3 if the number of active constraint sets in problem (8) is not large and Condition 2 is met, it is reasonable to use the ACS method; and

4 if Conditions 1 and 2 are not met but Condition 3 is met, one should use the ULB method.

Using the above methods a larger class of problems can be solved. However, there is an inherent drawback of all the methods discussed earlier. For many realistic problems, it is very difficult, if not impossible, to check the convexity (concavity) of $f$ and $g_{j}$. Even if it was possible to make such a check, the functions may turn out to be neither convex nor concave. Thus the above methods cannot guarantee 
the flexibility of most realistic CP's. To address this issue, we will now consider methods with a minimal dependence on knowledge of convexity (concavity) properties of the functions $f$ and $g_{j}$.

\section{MODIFICATION OF THE ULB METHOD}

We have developed two modifications of the ULB method, which will obtain a solution of TSOP with guaranteed flexibility for the case when Condition 3.2 is not met. The flexibility of the CP can be guaranteed if we can find global solutions to problem (17). In light of this, let us rewrite (17) and (19) in the form

$$
\max _{t \in T_{l}} g_{j}\left(d^{*}, z^{l *}, t\right) \leq 0 \quad l=1, \ldots, N_{k} ; j=1, \ldots, m
$$

These conditions are equivalent to

$$
g_{j}\left(d^{*}, z^{l *}, t\right), \quad \leq \quad 0 \quad j=1, \ldots, m, \quad \forall t \in T_{l}, l=1, \ldots, N_{k} ;
$$

The conditions in (24) mean that for each subregion $T_{l}(l=1, \ldots, N)$ we are able to find a vector of control variables $z^{l *}$, which guarantee satisfaction of all the constraints in (3). It should be noted that the dimensionality of problem (17) is less than the dimensionality of problem (9). However, finding the global maximum at each iteration is expected to be very computationally intensive. Subsequently, we suggest the following modification of the ULB method. First, let us consider the flexibility function $F_{1}$. In subregion $T_{l}$, we will use as upper bound for $h(d, t)$ the value

$$
\begin{aligned}
\bar{F}_{2 l} & =\min _{u, z \in Z} u \\
\max _{t \in T_{l}} U\left(g_{j} ; T_{l}\right) & \leq u, \quad j=1, \ldots, m,
\end{aligned}
$$

where $U\left(g_{j} ; T_{l}\right)$ is a concave overestimator of $g_{j}$ in $T_{l}$ with respect to $t$ satisfying the conditions

$$
U\left(g_{j} ; T_{l}\right) \geq g_{j}, \forall t \in T_{l} .
$$

If each $g_{j}$ is bilinear, then McCormick's (1976) technique can be used to construct $U\left(g_{j} ; T_{l}\right)$ whilst for general polynomial functions $g_{j}$, Sherali and Alameddine's (1992) linearization-reformulation approach is applicable.

Comparing problems (12) and (25) and taking into account (11), it is easy to obtain the following inequality

$$
\bar{F}_{2 i} \geq F_{2 i} \geq h(d, t)
$$


We will assume that $U\left(g_{j} ; T_{i}\right)$ satisfies the condition

$$
\lim _{r\left(T_{l}\right) \rightarrow 0} \max \left[U\left(g_{j} ; T_{l}\right)-g_{j}(d, z, t)\right]=0,
$$

where $r\left(T_{l}\right)$ is a measure of the size of $T_{l}$. This means that, as $T_{i}$ decreases in size, $U\left(g_{j} ; T_{i}\right)$ gets closer to $g_{j}$. Consequently $\bar{F}_{2 i}$ becomes a more accurate estimate of $h(d, t)$. In the limit we obtain (27) that

$$
\lim _{r\left(T_{i}\right) \rightarrow 0} \bar{F}_{2 i}=h\left(d, t_{i}\right)
$$

and $T_{i}$ reduces to a point $t_{i}$.

It is clear that the modification can be used if Condition 1 is satisfied. This modification is expected to be superior to the HG method since it does not require full enumeration. It is also expected that it will be superior to the SG method since it can be applied to a broader class of problems.

Let us revisit the ULB method for solving the TSOP. In order to obtain the upper bound $f^{U,(k)}$ of the objective function, we will solve the problem

$$
\begin{aligned}
\bar{f}_{3} & =\min _{d, z^{i}, z^{l}} \sum_{i \in I_{1}} w_{i} f\left(d, z^{i}, t^{i}\right) \\
g_{j}\left(d, z^{i}, t^{i}\right) & \leq 0, \quad i \in I_{1}, j=1, \ldots, m \\
\max _{t \in T_{l}} U\left[g_{j}\left(d, z^{l}, t\right) ; T_{l}\right] & \leq 0, \quad l=1, \ldots, N_{k}, j=1, \ldots, m
\end{aligned}
$$

and replace (17) by

$$
\max _{t \in T_{l}} U\left[g_{j}\left(d^{*}, z^{l *}, t\right) ; T_{l}\right]
$$

where $d^{*}, z^{l *}$ is a solution of problem (16). Of course we obtain an upper bound which is worse than the upper bound from (13). It is easy to see that if the following condition

$$
\max _{t \in T_{l}} U\left[g_{j}\left(d^{*}, z^{l *}, t\right) ; T_{l}\right] \leq 0
$$

is met, then condition (23) is met as well. Therefore, in the ULB method, we only need to replace (17) (i.e. maximization of each $g_{j}$ ) with the maximization of the concave overestimator of each $g_{j}$ (i.e. by (30)). Now consider the case when it is impossible to obtain an explicit expression for the state variable $x=x(d, z, t)$. For a calculation of an upper bound in this case it is necessary to solve problem (18) instead of problem (17). 
To formulate the equivalent of problem (30) we first replace each equality constraint in (18) with two inequality constraints which results in

$$
\begin{aligned}
& \max _{x, t \in T_{l}} \bar{g}_{j}\left(d^{*}, x, z^{l *}, t\right) \\
& \varphi\left(d^{*}, x, z^{l *}, t\right) \leq 0 \\
&-\varphi\left(d^{*}, x, z^{l *}, t\right) \leq 0 .
\end{aligned}
$$

Now problem (31) for calculating the upper bound will be equivalent to solving the problem

$$
\begin{aligned}
& \max _{x, t \in T_{l}} U\left[\bar{g}_{j}\left(d^{*}, x, z^{l *}, t\right)\right] \\
& L\left[\varphi\left(d^{*}, x, z^{l *}, t\right)\right] \leq 0 \\
& L\left[-\varphi\left(d^{*}, x, z^{l *}, t\right)\right] \leq 0
\end{aligned}
$$

where $L\left[\varphi\left(d^{*}, x, z^{l *}, t\right)\right]$ is a convex lower estimator of $f$ with respect to $t$.

Since the overestimator $U\left(g_{j} ; T_{i}\right)$ tends to $g_{j}$ as $T_{i}$ becomes infinitesimal, its use will not result in an increase in the number of iterative levels of the ULB method. Thus the computational complexity is not increased by the modification. We refer to the current modification as ULBG1. Suppose Condition 3.1 is not met for ULBG1, then it is easy to show that a solution of the TSOP guarantees the flexibility of the CP. In this case, we should note that the solution is in general a local minimum from the point of view of the objective function (4).

Let us consider a second modification ULBG2 to the ULB method. We have noted already that if the ULB method can obtain the solution of the TSOP then in many cases the solution will guarantee flexibility of the CP. However, we need an unambiguous answer with regard to flexibility. Thus, after obtaining the solution, we need to solve problem (17) using a global optimization method, specifically a branch and bound strategy. If condition (23) is met, then the solution guarantees $\mathrm{CP}$ flexibility. Otherwise the points $t_{j}^{l}$ at which condition (23) is violated are added to the corresponding sets of critical points $S_{21}$. In the computational experiments we have done so far, this procedure involves only one iteration in most of the cases.

\section{COMPUTATIONAL EXPERIMENTS}

In this section the proposed algorithms are applied to the optimization of a flowsheet consisting of a reactor and a heat exchanger (Fig. 1 from Halemane and Grossmann, 1983).

The reaction is assumed to be first-order exothermal of the type $\mathbf{A} \Longrightarrow \mathbf{B}$. The flow rate through the heat exchanger loop is adjusted to 


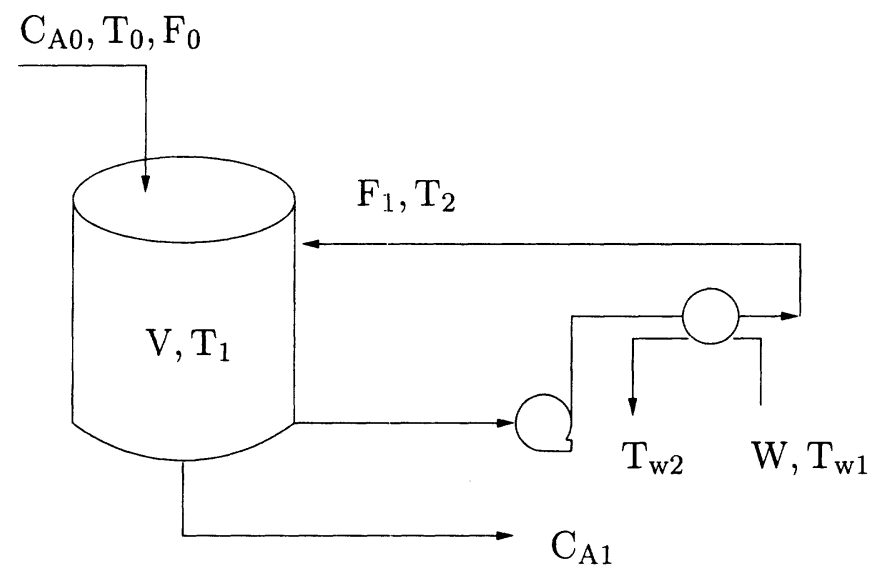

Figure 1 Flowsheet for example.

maintain the reactor temperature below $T_{1 \max }$ and to get a minimum of $90 \%$ conversion. The latter is given by conv $=\left(c_{A 0}-c_{A 1}\right) / c_{A 0}$. The performance equations of such a system are as follows

Reactor Material and Heat Balance

$$
\begin{aligned}
F_{0}\left(c_{A 0}-c_{A 1}\right) / c_{A 0} & =V k_{R} \exp \left(-E / R T_{1}\right) C_{A 1} \\
(-H) F_{0}\left(c_{A 0}-c_{A 1}\right) / c_{A 0} & =F_{0} c_{p}\left(T_{1}-T_{0}\right)+Q_{H E}
\end{aligned}
$$

Heat Exchanger Heat Balance and Design Equations

$$
\begin{aligned}
Q_{H E} & =F_{1} c_{p}\left(T_{1}-T_{2}\right)=c_{p w}\left(T_{w 2}-T_{w 1}\right) W \\
Q_{H E} & =A U \frac{\left(T_{1}-T_{w 2}\right)-\left(T_{2}-T_{w 1}\right)}{\ln \left\{\left(T_{1}-T_{w 2}\right) /\left(T_{2}-T_{w 1}\right)\right\}}
\end{aligned}
$$

where $F_{0}, T_{0}, C_{A 0}$ are the feed flow rate $\left([=] \mathrm{kgmolh}^{-1}\right)$, temperature of the feed $\left([=]^{\circ} K\right)$ and the concentration of the reactant in the feed ([=] $\mathrm{kgmolm}^{-1}$ ), respectively; $V, T_{1}, C_{A 1}$ are the values of the reactor volume $\left([=] \mathrm{m}^{3}\right)$, the reactor temperature $\left([=]^{\circ} \mathrm{K}\right)$ and the concentration of the reactant $\mathbf{A}$ in the product $\left([=] \mathrm{kgmolm}^{-3}\right) ; \mathrm{H}$ is the heat of the reaction $\left([=] \mathrm{kJ} \mathrm{kg}^{-1} \mathrm{~mol}^{-1}\right) ; F_{1}$ is the flow rate of the recycle ([=] $\left.\mathrm{kgmolh}^{-1}\right) ; T_{2}$ is the recycle temperature; $c_{p}=167.4 \mathrm{~kJ} \mathrm{kgmol}^{-1}$ and $c_{p w}=4.19 \mathrm{~kJ} \mathrm{kgmol}^{-1}$ are the heat capacities of the recycle mixture and the cooling water, respectively. $T_{w 1}, T_{w 2}, W$ are the inlet and outlet temperatures and the flow rate $\left(k g h^{-1}\right)$ of the cooling water respectively. $A\left([=] m^{2}\right)$ is the heat transfer area of the heat exchanger and $U([=]$ $\left.k J m^{-2} h^{-1} k^{-1}\right)$ is the overall heat transfer coefficient. 
In this problem the following constraints apply

$$
\begin{aligned}
- \text { conv }+0.9 & \leq 0 \\
\text { conv }-1 & \leq 0 \\
-\left(T_{2}-T_{w 1}\right)+11.1 & \leq 0 \\
T_{2}-389 & \leq 0 \\
-T_{2}+311 & \leq 0 \\
T_{2}-T_{1} & \leq 0 \\
-T_{w 2}+T_{w 1} & \leq 0 \\
311 & \leq T_{1} \leq 389 \\
301 & \leq T_{w 2} \leq 355 .
\end{aligned}
$$

The objective function of the original optimization problem is of the form

$$
F=691.2 V^{0.7}+873.6 A^{0.6}+1.76 W+7.056 F_{1} .
$$

The design variables are $V$ and $A$. The control variables are $T_{1}$ and $T_{w 2}$. The vector of state variables is $\left[C_{A 1}, T_{2}, F_{1}, W\right]$. The vector of uncertain parameters is $t=\left[F_{0}, T_{0}, T_{w 1}, k_{R}, U\right]$. Finally, the uncertainty region $T$ is given by

$$
T(\gamma)=\left[t_{i}^{N}\left(1-\gamma \delta t_{i}\right) \leq t_{i} \leq t_{i}^{N}\left(1+\gamma \delta t_{i}\right)\right]
$$

where $t_{i}^{N}=(45,333,300,9.8,1635)$ is the nominal value of the uncertain parameters, $\gamma$ is the parameter to determine the size of the uncertain parameter range, and $\delta t_{i}=(0.1,0.02,0.03,0.1,0.1)$ is a deviation fraction. The case $\gamma=1$ was investigated by Halemane and Grossmann (1983) and Ostrovski and Volin (1994).

After elimination of dependent (i.e. state) variables $C_{A 1}, T_{2}, F, w$ using equations (32) and (33), we obtain

$$
\begin{aligned}
\text { conv } & =\frac{V k_{R} c_{A 0} \exp \left(-E / R T_{1}\right)}{F_{0}+V k_{R} c_{A 0} \exp \left(-E / R T_{1}\right)} \\
T_{2} & =\frac{2(-H) F_{0} \operatorname{con} v}{A U}-\frac{2 F_{0} c_{p}\left(T_{1}-T_{0}\right)}{A U}-\left(T_{1}-T_{w 2}\right)+T_{w 1}
\end{aligned}
$$

Substituting the expressions into the constraints (34) to (40), we obtain explicit expressions for $g_{j}$ with respect to the control variables and the uncertain parameters. These expressions contain linear, bilinear and trilinear terms with respect to the uncertain parameters. In Table 1, we give the approximation points from (Halemane and Grossmann,1983). Here, $N, L, U$ designate nominal, lower and upper bounds. 


\begin{tabular}{cccccc}
\hline & $F_{0}$ & $T_{0}$ & $T_{w 1}$ & $K_{0}$ & $\mathrm{U}$ \\
\hline$t^{1}$ & $\mathrm{~N}$ & $\mathrm{~N}$ & $\mathrm{~N}$ & $\mathrm{~N}$ & $\mathrm{~N}$ \\
$t^{2}$ & $\mathrm{~L}$ & $\mathrm{~L}$ & $\mathrm{~L}$ & $\mathrm{~L}$ & $\mathrm{U}$ \\
$t^{3}$ & $\mathrm{U}$ & $\mathrm{U}$ & $\mathrm{U}$ & $\mathrm{U}$ & $\mathrm{L}$ \\
$t^{4}$ & $\mathrm{U}$ & $\mathrm{U}$ & $\mathrm{L}$ & $\mathrm{U}$ & $\mathrm{L}$ \\
$t^{5}$ & $\mathrm{U}$ & $\mathrm{U}$ & $\mathrm{U}$ & $\mathrm{L}$ & $\mathrm{L}$ \\
\hline
\end{tabular}

Table 1 Approximation points: N - nominal value, L - lower bound, U - upper bound

We solved the problem for different numbers of approximation points and different sizes of the uncertainty region. In Table 2 we present results for (a) the nominal values of the uncertain parameters and (b) different sizes of the uncertainty region. In (9) we used five approximation points from Table 1. In the last column, the number of iterations used in the ULB method is presented.

\begin{tabular}{lccccc}
\hline & $\gamma$ & $f_{1}$ & $\mathrm{~V}$ & $\mathrm{~A}$ & \# Iter \\
\hline Optimization under Nominal & - & 9003.62 & 5.42 & 5.21 & 1 \\
values of Uncertain Parameters & & & & & \\
Optimization under Uncertainty & 1.0 & 10670.7 & 6.63 & 7.77 & 1 \\
Optimization under Uncertainty & 1.25 & 11187.5 & 6.97 & 8.57 & 2 \\
Optimization under Uncertainty & 1.50 & 11776.5 & 7.34 & 9.45 & 5 \\
Optimization under Uncertainty & 1.75 & 12413.2 & 7.72 & 10.42 & 5 \\
\hline
\end{tabular}

Table 2 Results for different sizes of uncertainty region

In Table 3 we present TSOP results for different numbers $\left(I_{5}\right)$ of approximation points for $\gamma=1$. We solved two variants corresponding to five approximation points and the first three approximation points from Table 1. If we compare the results of nominal optimization and optimization under uncertainty for $\gamma=1$ and five approximation points, we conclude that it is necessary to increase the reactor volume by $25 \%$ and the heat exchange area by $22 \%$. However, the bilinear and trilinear terms are sources of multiextremality in problem (17). As such we cannot guarantee flexibility of the process (i.e. Condition 3.2 cannot be met).

In light of this we solved the problem using the ULBG1 and ULBG2 methods. The ULBG1 method requires construction of concave overesti- 


\begin{tabular}{lccccc}
\hline & Is & $f_{1}$ & $\mathrm{~V}$ & $\mathrm{~A}$ & \# Iter \\
\hline Optimization under Nominal & - & 9003.62 & 5.42 & 5.21 & 1 \\
values of Uncertain Parameters & & & & & \\
Optimization under Uncertainty & 5 & 10670.7 & 6.63 & 7.77 & 1 \\
Optimization under Uncertainty & 3 & 10033.7 & 6.05 & 7.67 & 1 \\
\hline
\end{tabular}

Table 3 Results for $\gamma=1$ and different numbers of approximation points

mators $U\left(g_{j}\right)$ of $g_{j}$. Since the latter contain bilinear and trilinear terms, we must construct overestimators for these terms. For bilinear terms we used McCormick's (1983) expressions for overestimators and for trilinear terms we used as overestimators expressions from (Maranas and Floudas ,1995) (an extension of the overestimator for a bilinear term). We solved for $\left(I_{5}=5\right)$ and $\gamma=(1,1.25,1.5)$. Results are given in Table 4. In addition, we solved for $\gamma=1$ and $I_{5}=(5,3)$. Results are given in Table 5.

\begin{tabular}{lccccc}
\hline & $\gamma$ & $f_{1}$ & $\mathrm{~V}$ & $\mathrm{~A}$ & \# Iter \\
\hline Optimization under Nominal & - & 9003.62 & 5.42 & 5.21 & 1 \\
values of Uncertain Parameters & & & & & \\
Optimization under Uncertainty & 1.0 & 10670.7 & 6.63 & 7.77 & 1 \\
Optimization under Uncertainty & 1.25 & 11187.5 & 6.97 & 8.57 & 1 \\
Optimization under Uncertainty & 1.50 & 11776.5 & 7.34 & 9.45 & 1 \\
\hline
\end{tabular}

Table 4 Results with the ULBG1 method

\begin{tabular}{lccccc}
\hline & Is & $\mathrm{f}$ & $\mathrm{V}$ & $\mathrm{A}$ & \# Iter \\
\hline Optimization under Nominal & - & 9003.62 & 5.42 & 5.21 & 1 \\
values of Uncertain Parameters & & & & & \\
Optimization under Uncertainty & 5 & 10670.7 & 6.63 & 7.77 & 1 \\
Optimization under Uncertainty & 3 & 10033.7 & 6.05 & 7.67 & 1 \\
\hline
\end{tabular}

Table 5 Results with the ULBG2 method

Comparing the results from Table 4 with those of Table 2 and the results from Table 5 with those of Table 3 , show that we can ensure 
flexibility of the process. It is interesting to note that the use of the overestimators does not increase the number of iterations.

In the ULBG2 method, we must take the values of design variables obtained by the ULB method and use them as initial points for global optimization of problems (17). For calculation of an upper bound of the functions $g_{j}$ we used the overestimators, which were constructed by the technique described above. We solved the TSOP problem for the case when five approximation points are used and $\gamma=(1,1.25,1.5)$. Global optimization of all the constraints for all the cases showed that the solutions found by the ULB method are the global maxima of $g_{j}$ with respect to the uncertain parameters. Consequently, the solutions obtained by the ULB method guarantee flexibility of the process. It is interesting to note that in all the cases the branch and bound global optimization method found global maxima in one iteration.

\section{DISCUSSIONS AND CONCLUSIONS}

The aim of solving the two step optimization problem consists in determining optimal design margins guaranteeing the preservation of capacity for the operation of chemical processes (flexibility of chemical processes) in spite of model uncertainties at the design stage, and process uncertainties at the operation stage. The design margins obtained can, however, be used in practice only if the solution of the two-stage optimization problem under uncertainty (TSOP) corresponds to the global solution of the flexibility problem. We have therefore carried out a comparative analysis of three deterministic methods of flexibility analysis and optimization of chemical processes under uncertainty.

Our analysis showed that the methods discussed in this paper supplement each other to some degree. Given this range of tools, one can solve a wide class of optimization problems. However there is an inherent drawback of the methods. For many real problems, it is very difficult to check the convexity (concavity) of the constraint and objective functions. Even if it was possible to carry out such a check, the functions may turn out to be neither convex nor concave. As a result, there is a need to develop methods that have minimal requirements on convexity (concavity) of the constraint and objective functions.

We have developed two significant modifications of the ULB method which permit us to obtain the solution of the TSOP guaranteeing flexibility of $\mathrm{CP}$ under a single condition, namely the convexity of the constraint functions $g_{j}(d, z, t)$ in $z$. Any solution obtained by the algorithm will guarantee flexibility of the $\mathrm{CP}$ even though the solution may correspond to a local minimum of the TSOP. 


\section{References}

[1] M.S. Bazaraa, H.D. Sherali and C.M. Shetty (1993), Non-linear Programming, Theory and Algorithms, John Wiley and Sons, New York.

[2] L.T. Biegler, I.E. Grossmann and A.W. Westerberg (1997), Systematic Methods of Chemical Process Design, Prentice Hall.

[3] I.E. Grossmann and C.A. Floudas (1987), Active constraint strategy for flexibility analysis in chemical processes, Comp. \&6 Chem. Eng., 11, pp. 675-693.

[4] K.P. Halemane and I.E. Grossmann (1983), Optimal process design under uncertainty, AIChE Journal, 29, pp. 425-433.

[5] U. Kabatek and R.E. Swaney (1992), Worst-case identification in structured process systems, Comp. \& Chem. Eng., 16, pp. 10631071.

[6] G.P. McCormick (1976), Computability of global solutions to factorable nonconvex programs. Part I - convex underestimating problems, Mathematical Programming, 10, pp. 147-175.

[7] G.M. Ostrovsky, Yu.M. Volin, I.E. Barit and M.M. Senyavin (1994), Flexibility analysis and optimization of chemical plants under uncertainty, Comp \& Chem. Eng, 18, pp. 755-767.

[8] G.M. Ostrovsky, Yu.M. Volin, E.I. Barit and M.M. Senyavin (1997), An approach to solving a two step optimization problem, Comp. E6 Chem. Eng., 21, pp. 317-325.

[9] E.N. Pistikopoulos and I.E. Grossmann (1989), Optimal retrofit design for improving process flexibility in non-linear systems-1, Fixed degree of flexibility, Comp. \& Chem. Eng., 12, pp. 1003-1016.

[10] E.N. Pistikopoulos and M.G. Ieraptritou (1995), Novel approach for optimal process design under uncertainty, Comp \& Chem. Eng., 19, pp. 1089-1110.

[11] H.D. Sherali and Tunchbilek (1992), A global optimization algorithm for polynomial programming programs using reformulationlinearization technique, Journal of Global Optimization, 2, pp. 101112.

[12] R.E. Swaney and I.E. Grossmann (1985), An index for operational flexibility in chemical process design, AIChE Journal, 31, pp. 621630 . 